\title{
Multiple Unmanned Aerial Vehicles Cooperative Combat Task Allocation Method Based on Receding Horizon Control
}

\author{
Xia Chen ${ }^{1}$, Fuyu Zhao ${ }^{2}$ and Liang Zhao ${ }^{3}$ \\ 1, 2, 3 School of Automation, Shenyang Aerospace University 110136, Shen Yang, \\ China; \\ xiachen1108@163.com \\ 503171379@qq.com \\ 944133357@qq.com
}

\begin{abstract}
For multiple unmanned aerial vehicles (multi-UAV) task allocation, a new method is given. Firstly, the capability function of UAV is established, and the calculation method of multi-UAV cooperative combat position is presented through the capability function of Jacobian matrix. The UAV's value income index function, damage index function, voyage index function and multi-UAV cooperative task allocation model are established further. The maneuvering decision making problem is modeled as the optimal control problem by using Receding Horizon Control (RHC). Then the optimal decision-making method of multi-UAV cooperative combat task allocation is gotten by the discretization of time and space in the whole process of decision-making and the calculation of the optimal maneuver strategy piecewise. Finally, the simulation experiment is conducted, the result of simulation shows that the method can get more profit and damage efficiency of the targets, and improve the capability of multi-UAVs cooperative combat.
\end{abstract}

Keywords: Multiple unmanned aerial vehicles; capability function; collaborative task allocation; Jacobian Matrix; Receding Horizon Control (RHC)

\section{Introduction}

The aircraft has played an important role in the modern war, and it has become the most useful and the most suitable weapon in the wide range of war all over the world. The research on the combat effectiveness of aircraft can not only help to improve the aircraft performance and promote the development of tactics, but also have the great significance to strengthen the national defense of our country. Although the single combat has certain advantages, it is far from satisfaction of combat with the complication of today's battlefield. The multi-UAV cooperative combat can cover the shortage of single combat. Compared with single combat the multi-UAV cooperative combat has advantages in the aspect of target detection, target attack and comprehensive fire. Therefore, the multi-UAV cooperative combat will be the main combat mode in the future, and it can enhance the combat effectiveness significantly ${ }^{[1]}$. However, one of the key issues is the problem of proper coordination between the multi-UAV in a task ${ }^{[2]}$. So it's a hot topic that how to make a reasonable decision which UAVs could accomplish complex coordination task in the field of UAV research ${ }^{[3]}$. At present, the research that the air combat task allocation of multiple unmanned aerial vehicles cooperative attack has made some valuable research results ${ }^{[4-7]}$.In [4], the UAV cooperative combat task allocation model was proposed which is based on the bi-level programming theory. In [5], a mathematical model of multiple vehicles coordinated target assignment was built which was based on the evaluation of vehicle residual ability. In [6], the multiple UCAV cooperative task assignment model for attacking the ground targets was built and the particle swarm optimization (PSO) 
algorithm for solving such a problem was proposed, based on proper task assignment solution to PSO particle mapping. In [7], according to multitask allocation problem of multi-shipboard UAV cooperative attack function model of the overall allocation performance was established based on task allocation of benefits and costs through reasonable assumptions to abstract and simplify the problem. In [8], the ACBAA (asynchronous consensus-based auction algorithm) algorithm was improved, and the method of air-combat decision-making for multiple UAV cooperatively attacking multiple targets in uncertain environment was proposed. In [9], the multistage influence diagram was used to solve the maneuvering decision, but its model was complex and the calculating amount is large, so it was difficult to meet the real-time requirements of decision making. However, in the prior literature, both the cooperative combat ability of multi-UAV and the value of the capability which the target was required were not considered in the model.

Currently, the research of considering the capability function of robot in the task allocation of multi-robot has attracted the attention of scholars ${ }^{[10,11]}$.In [10], a new method of the task allocation of multi-robot system based on the utility function of robot was proposed. In [11], a task allocation of multi-robot system based on the utility function was proposed, the mathematical model of the utility function was established based on the vector of the robot's capability and the vector of capability that the subtasks required, and then make the task assignment according to the size of the utility function. But so far, the papers about the task assignment related to the capability function of the multi-UAV cooperative combat task haven't been reported in the literature. This paper proposes the method of calculating the positions of multi-UAV cooperative combat and the method of multi-UAV cooperative combat task allocation. The Receding Horizon Control (RHC) is a control technology which is based on on-line calculation and can solve the system optimal control problem by rolling optimization. In the process of optimization the real-time system information can be used to feedback compensation. The robustness of the system can be enhanced and the solution is relatively simple by using RHC. So we use the RHC to model the maneuvering decision making problem as the optimal control problem. The optimal decision-making method of multi-UAV cooperative combat task allocation is gotten by the discretization of time and space in the whole process of decision-making and the calculation of the optimal maneuver strategy piecewise.

\section{The Calculation of the UAV's Capability Function and the Combat Range of Multi-UAV Cooperative}

\subsection{The Establish of Capability Function of the UAV}

The capability function reflects the capability of UAV attack to the target, and it's based on the requirements and form of the task. The capability function is related to the factors such as the distance and azimuth between UAVs and targets. The capability function of multi-UAV is the sum of the capability function of the UAV. In order to simplify the analysis, we assume that the capability function is only related to the distance between UAV and the target, when the distance between the UAV and the target meet the requirements, the maximum capability value of the UAV will be achieved. The information of the position of UAV is stored in the $n \times 3$ matrix, $n$ indicates the number of $\mathrm{UAV}$. The information of the position of targets is stored in the $n \times 3$ matrix, $m$ indicates the number of targets. The initial position matrix of the UAV and target is as follow. 


$$
P_{\mathrm{UAV}}=\left[\begin{array}{ccc}
x_{U 1} & y_{U 1} & z_{U 1} \\
x_{U 2} & y_{U 2} & z_{U 2} \\
\mathrm{M} & \mathrm{M} & \mathrm{M} \\
x_{U n} & y_{U n} & z_{U n}
\end{array}\right] \quad P_{\text {target }}=\left[\begin{array}{ccc}
x_{T 1} & y_{T 1} & z_{T 1} \\
x_{T 2} & y_{T 2} & z_{T 2} \\
\mathrm{M} & \mathrm{M} & \mathrm{M} \\
x_{T m} & y_{T m} & z_{T m}
\end{array}\right]
$$

The distance $d_{i j}$ between the $i$-th UAV and the $j$-th target is described as

$$
d_{i j}=\sqrt{\left(x_{U i}-x_{T j}\right)^{2}+\left(y_{U i}-y_{T j}\right)^{2}+\left(z_{U i}-z_{T j}\right)^{2}}
$$

Assume that the combat radius of $\mathrm{UAV}$ is $D_{i}$, the maximum capability of the function is 1 , the capability function of the $i$-th target is described as $B_{i}$,

$$
B_{i}=\left\{\begin{array}{c}
D_{i} / d_{i j}, d_{i j} \geq D_{i} \\
d_{i j} / D_{i}, d_{i j}<D_{i}
\end{array}\right.
$$

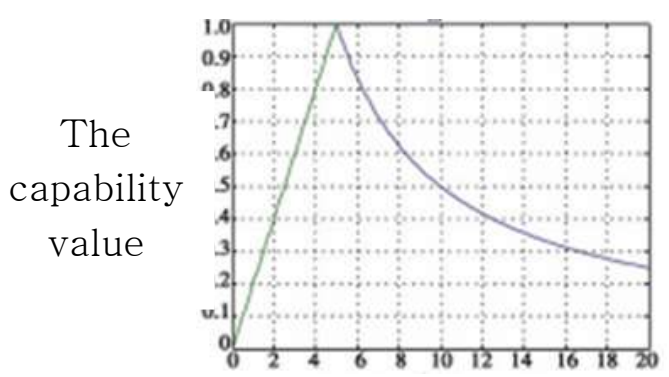

The distance/km

Figure 1. The Curve of the Capability Function of UAV

Assume that the radius of tactical reconnaissance of UAV is $4.5 \mathrm{~km}$, in Figure 1, the capability decreases with the increase of the distance, when the distance between the UAV and the target is more than $4.5 \mathrm{~km}$, and the capability increases with the decrease of the distance, when the distance between the UAV and the target is less than $4.5 \mathrm{~km}$. Only when the distance between the UAV and the target is more than $4.5 \mathrm{~km}$, the maximum capability value of the UAV will be achieved.

\subsection{The Jacobian Matrix}

For the UAV, in order to complete the task more effectively, we need to analyze the impact that the changes of the UAV's position on the ability that the target is required. So the Jacobian matrix of the capability function is established as follow.

$$
J_{J a c o b i}=\left[\begin{array}{cccccc}
\frac{\partial B_{1}}{\partial x_{U 1}} & \frac{\partial B_{1}}{\partial y_{U 1}} & \frac{\partial B_{1}}{\partial z_{U 1}} \mathrm{~L} & \frac{\partial B_{1}}{\partial x_{U n}} & \frac{\partial B_{1}}{\partial y_{U n}} \frac{\partial B_{1}}{\partial z_{U n}} \\
\mathrm{M} & \mathrm{M} & \mathrm{M} & \mathrm{M} & \mathrm{M} & \mathrm{M} \\
\frac{\partial B_{m}}{\partial x_{U 1}} & \frac{\partial B_{m}}{\partial y_{U 1}} & \frac{\partial B_{m}}{\partial z_{U 1}} \mathrm{~L} & \frac{\partial B_{m}}{\partial x_{U n}} & \frac{\partial B_{m}}{\partial y_{U n}} \frac{\partial B_{m}}{\partial z_{U n}}
\end{array}\right]
$$

Besides, 


$$
\begin{aligned}
& \frac{\partial B_{j}}{\partial x_{U i}}=\left\{\begin{array}{c}
\frac{-\mathrm{D}_{i} * x_{U i}}{\left[\left(x_{U i}-x_{T j}\right)^{2}+\left(y_{U i}-y_{T j}\right)^{2}+\left(z_{U i}-z_{T j}\right)^{2}\right]^{3 / 2}}, d_{i j}>\mathrm{D}_{i} \\
\frac{x_{R i} *\left[\left(x_{U i}-x_{T j}\right)^{2}+\left(y_{U i}-y_{T j}\right)^{2}+\left(z_{U i}-z_{T j}\right)^{2}\right]^{-1 / 2}}{\mathrm{D}_{i}}, d_{i j}<\mathrm{D}_{i}
\end{array}\right. \\
& \frac{\partial B_{j}}{\partial y_{U i}}=\left\{\begin{array}{c}
\frac{-\mathrm{D}_{i} * y_{U i}}{\left[\left(x_{U i}-x_{T j}\right)^{2}+\left(y_{U i}-y_{T j}\right)^{2}+\left(z_{U i}-z_{T j}\right)^{2}\right]^{3 / 2}}, d_{i j}>\mathrm{D}_{i} \\
\frac{y_{R i} *\left[\left(x_{U i}-x_{T j}\right)^{2}+\left(y_{U i}-y_{T j}\right)^{2}+\left(z_{U i}-z_{T j}\right)^{2}\right]^{-1 / 2}}{\mathrm{D}_{i}}, d_{i j}<\mathrm{D}_{i}
\end{array}\right. \\
& \frac{\partial B_{j}}{\partial z_{U i}}=\left\{\begin{array}{c}
\frac{-\mathrm{D}_{i} * z_{U i}}{\left[\left(x_{U i}-x_{T j}\right)^{2}+\left(y_{U i}-y_{T j}\right)^{2}+\left(z_{U i}-z_{T j}\right)^{2}\right]^{3 / 2}}, d_{i j}>\mathrm{D}_{i} \\
\frac{Z_{R i} *\left[\left(x_{U i}-x_{T j}\right)^{2}+\left(y_{U i}-y_{T j}\right)^{2}+\left(z_{U i}-z_{T j}\right)^{2}\right]^{-1 / 2}}{\mathrm{D}_{i}}, d_{i j}<\mathrm{D}_{i}
\end{array}\right.
\end{aligned}
$$

\subsection{The Calculation of the Combat Position of Multi-UAV Cooperative}

The capability function is determined by the position of the UAV, and the relationship between the position and the change of the capability function as follow.

$$
\left[\begin{array}{ll}
\dot{B}_{1} \mathrm{~L} & \dot{B}_{m}
\end{array}\right]^{\mathrm{T}}=J_{J a c b i} *\left[\begin{array}{ccccccc}
\dot{x_{U 1}} & \dot{\mathrm{y}_{U 1}} & \dot{z_{U 1}} & \mathrm{~L} & \dot{x_{U n}} & \dot{\mathrm{y}_{U n}} & \dot{z}_{U n}
\end{array}\right]^{\mathrm{T}}
$$

Assume that the ideal capability that the $\operatorname{target} j$ needed is $B_{j}^{d}$, and the ideal change of the capability function is equal to the difference between the current capability value $B_{j}$ and the ideal capability value $B_{j}^{d}$. To pre-allocate the UAV according to the allocation plan of UAVs to get the corresponding $B_{l}^{1}, B_{l}^{2}, \ldots B_{l}^{m} \quad\left(B_{l}^{m}\right.$ is the capability value of the $m$-th target in the $l$ - th allocation plan) as shown in (1.9)

$$
\left[\begin{array}{ccccccc}
\dot{x_{U 1}} & \dot{\mathrm{y}_{U 1}} & \dot{z_{U 1}} & \mathrm{~L} & \dot{x_{U n}} & \dot{\mathrm{y}_{U n}} & \dot{z}_{U n}
\end{array}\right]^{\mathrm{T}}=J_{\text {Jacobi }}^{+} *\left(\left[\begin{array}{c}
B_{1}^{d} \\
\mathrm{M} \\
B_{m}^{d}
\end{array}\right]-\left[\begin{array}{c}
B_{l}^{1} \\
\mathrm{M} \\
B_{l}^{m}
\end{array}\right]\right)
$$

$J_{\text {Jacobi }}^{+}$is the pseudo inverse of the Jacobian matrix $J_{\text {Jacobi }}$ in (2.9).

$$
J^{+}=J^{\mathrm{T}}\left(J J^{\mathrm{T}}\right)^{-1}
$$

$J^{+}$represents the pseudo inverse and $J^{\mathrm{T}}$ represents the transposition of Jacobian matrix.

The positions of UAV need to be updated if the cooperative combat of multi-UAV can't reach the ideal capability. To get the ideal changes $\underset{x_{U i}}{\mathrm{~g}}, y_{U i}^{\mathrm{g}}$ and $z_{U i}^{\mathrm{g}}$ of the positions of $\mathrm{UAV}$, according to (2.9), and the time step is $\Delta t$, that we can get the new positions of UAV at the $(k+1)$-th time step, and the equation that the UAV update their positions is shown as (2.11). 


$$
\left[\begin{array}{c}
x_{U i}[k+1] \\
y_{U i}[k+1] \\
z_{U i}[k+1]
\end{array}\right]=\left[\begin{array}{c}
x_{U i}[k] \\
y_{U i}[k] \\
z_{U i}[k]
\end{array}\right]+\left[\begin{array}{c}
\dot{x_{U i}} \\
\dot{y_{U i}} \\
\dot{z_{U i}}
\end{array}\right] * \Delta t
$$

\subsection{The Calculation of the Range of Multi-UAV Cooperative Combat}

We can get the new value of the capability of UAV by bringing the updated position coordinates into (2.2) and (2.3), and update the position of UAV according to (2.1) to (2.11) until reach the ideal value of the capability $B_{j}^{d}$. Then we can get all of the UAVs information of positions $\left(\mathrm{x}_{U i}^{l 0}, y_{U i}^{l 0}, z_{U i}^{l 0}\right),\left(\mathrm{x}_{U i}^{l 1}, y_{U i}^{l 1}, z_{U i}^{l 1}\right), \ldots,\left(\mathrm{x}_{U i}^{l \mathrm{~g}}, y_{U i}^{l \mathrm{~g}}, z_{U i}^{l \mathrm{~g}}\right),(i=1,2, \ldots, n)$ in the $g$ time steps under the $l$-th allocation plan, and get the range of UAV of every strategies. The calculation of range is shown as (2.12).

$$
D_{i j}=\sum_{k=0}^{g-1} \sqrt{\left.\left(x_{U i}^{l(k+1)}-x^{l}\right)_{U i}^{2}+y_{U i}^{(+k 1)}-y\right)_{U}^{2 k}\left(+z_{U i}^{+(l) k 1}\right) z^{2}}
$$

\section{The Establishment of the Multi-UAV Cooperative Task Allocation Model}

When making multi-UAV task allocation, the main factors need to be considered are the following two aspects. One is the attack earnings and the other is the cost in the process of attacking targets. The cost of our side can be consisted by two parts, one is the cost of flight, and the other is the damage cost caused by the antiaircraft fire of targets. Assuming that the number of UAV is $n$, and the number of target is $m$.

\subsection{The Index Function of the Target Value Income}

The target value income refers to the loss that our UAVs bring to the targets. In the process of the cooperative combat of multi-UAV, the benefit of the target value is an important indicator to be considered. Assuming that the damage probability matrix of our UAVs to the targets is $P=\left[\begin{array}{lllll}P_{1 j} & P_{2 j} & \cdots & P_{m j}\end{array}\right]$, so the Kill probability that the $i$-th UAV to the target is as follow.

$$
P_{i j}=1-\left(1-\eta_{i}\right)^{n_{i}}
$$

$\eta_{i}$ is the probability that the antiaircraft fire $j$ of the target destroyed by an UAV during $t_{k}<t<t_{k+1}$. To consider the closer the distance between an antiaircraft fire and an UAV, the bigger the $\eta_{i}$, that:

$$
\eta_{i}=\left\{\begin{array}{lll}
\left(1-d_{i j}\right. & u_{m} & \partial_{m} \quad, d_{i j}<u_{m} \\
0, & d_{i j}>u_{m}
\end{array}\right.
$$

$n_{i}$ is the number of weapons that carried by the $i$-th UAV, and the attack of an UAV to a target is an independent event. $d_{i j}$ is the distance between the $i$-th UAV and the $j$-th target, $u_{m}$ is the effective firing range of the UAV, and $\partial_{m} \in[0,1]$ is the destroy effectiveness of the UAV. Assuming that the value set of the target 
is $\left\{v_{1}^{d}, v_{2}^{d}, \mathrm{~L}, v_{j}^{d}, \mathrm{~L}, v_{m}^{d}\right\}$, and $v_{\max }^{d}$ is the maximum of the income, that the income index function $R_{i j}^{1}$ is as follow.

$$
R_{i j}^{1}=\frac{v_{j}^{d} * p_{i j}}{v_{\max }^{d}}
$$

If there are $q \mathrm{UAV}$ s attacking the $j$-th target cooperatively at the same time, that index function of the target value income $R_{j}^{q}$ is as follow.

$$
R_{j}^{q}=\frac{v_{j}^{d} *\left[1-\prod_{i=1}^{q}\left(1-p_{i j}\right)\right]}{v_{\max }^{d}}
$$

\subsection{The Index Function of Damage Price}

The damage price refers to the loss that the targets bring to our UAVs. The attack of a target to an UAV is an independent event, $P_{i j}^{\prime}(q+1)$ is the probability that the $i$-th UAV destroyed by the $j$-th target during $t_{k}<t<t_{k+1}$, so the damage probability that the $\mathrm{j}$-th target to the $i$-th $\mathrm{UAV}$ is as follow.

$$
P_{i j}^{\prime}(q+1)=1-\prod_{n=1}^{M_{n}}\left(1-\delta_{n}(q+1)\right)
$$

$M_{n}$ is the number of the target's antiaircraft fire, $\delta_{n}(q+1)$ is the probability that the $i$-th UAV destroyed by the $j$-th antiaircraft fire during $t_{k}<t<t_{k+1}$. To consider the closer the distance between an antiaircraft fire and an UAV, the bigger the $\delta_{n}(q+1)$, that:

$$
\delta_{n}(q+1)=\left\{\begin{array}{l}
\left(1-d_{i j} / e_{m}\right) \theta_{m}, \quad d_{i j}<e_{m} \\
0, \quad d_{i j}>e_{m}
\end{array}\right.
$$

$d_{i j}$ is the distance between the $i$-th UAV and the $j$-th target, $e_{m}$ is the effective firing range of the target, $\theta_{m} \in[0,1]$ is the destroy effectiveness of the target, $v_{a}^{i}$ is the value of the $i$-th $\mathrm{UAV}$, that the index function of damage price $C_{1}$ is as follow.

$$
C_{1}=\frac{v_{a}^{i} * p^{\prime}{ }_{i}}{v_{a \mathrm{max}}}
$$

If q UAVs attack the $j$-th target cooperatively, that the index function of the damage price is as follow.

$$
C_{j}^{q}=\frac{\frac{1}{q} \sum_{i=1}^{q} v_{a}^{i} * p^{\prime}}{v_{a \max }}
$$

\subsection{The Cost of Flight Route}

The cost of flight route is positively related the path of the flight, and the cost of flight route is as follow.

$$
C_{2}=\frac{D_{i j}}{D_{\max }} * x_{i j}
$$

In which, $D_{i j}$ is the actual flight distance of the $i$-th $\mathrm{UAV}$, and $D_{\max }$ is the 
maximum actual flight distance of all the UAVs.

If $q$ UAVs attack the $j$-th target cooperatively, that the index function of the cost of flight route is as follow.

$$
C_{2}=\frac{\frac{1}{q} \sum_{i=1}^{q} D_{i j}}{D_{\max }}
$$

\subsection{The Overall Earning Model of the Multi-UAV Cooperative Combat Task}

The purpose of the task allocation is to get the maximum value profit and pay the minimum cost by attacking targets under the condition that the scheduled tasks can be accomplished by the formation of multi-UAV. Besides, the importance of every index is different, so the weight is different. The overall earning model of the multi-UAV cooperative combat task is as follow.

$$
L=w_{1} * R-w_{2} * C_{1}-w_{3} * C_{2}
$$

s.t.

$$
\begin{gathered}
\sum_{j=1}^{m} x_{i j} \leq 1 \\
\sum_{i=1}^{n} x_{i j} \leq h \\
w_{1}+w_{2}+w_{3}=1
\end{gathered}
$$

$w_{1}, w_{2}$ and $w_{3}$ are the weight of the benefit of the target value, the damage price and the cost of flight route. The formula (3.11a) means that every UAV can be allocated to one target at most, and the formula (3.11b) means that the number of the UAV to attack a target cooperatively is $h$ at most.

\section{The RHC and its Numerical Solution}

\subsection{The RHC Method}

In the RHC, the decision of the control variable $u$ is made at the discrete time $t=k \Delta t, k$ is the step counter, and $\Delta t$ is the interval of decision time. The state of $t_{k}$ is denoted by $x\left(t_{k}\right)$, and we can get the optimal open loop control $u *\left(x_{\left(t_{k}\right)}\right)$ by the following model which is on the time domain $t \in\left[t_{k}, t_{k+T}\right]$.

$$
\begin{array}{ll}
\dot{L}_{k}(u)= & \int_{t_{k}}^{t_{k}+T} L(x, u, t) d t+V\left(x\left(t_{k}+T\right), t_{k}+T\right) \\
\text { s.t. } & \stackrel{\mathrm{g}}{x}=f(x, u, t), x\left(t_{k}\right)=x_{k} \\
& g(x, u, \stackrel{\mathrm{g}}{u}) \leq 0 \\
& h\left(x\left(t_{k}+T\right)\right)=0
\end{array}
$$

The integral part is the accumulated value of the indicator function $L$ on the planning time domain $T(T>\Delta t), V(\cdot)$ is value function which is used to approach the optimal price from the optimal price to the final state.

In order to make the current state of measured value as the initial conditions, to 
solve the problem as the Euler-Lagrange trajectory optimization, and calculate the optimal control solution $u^{*}$ online, then carrying out the control $u^{*}$ in time domain $T$ until the system gets the new state measurements and making the new state measurements as the new initial condition. Repeat the above process and calculate the optimal control solution of the finite time domain until meeting the requirements, so we can get a group of state feedback control law. The RHC method can avoid the dimension disaster of Hamilton-Jacobi Bellman (HJB).

\subsection{The Numerical Solution of RHC}

The general solution for the optimal control problem is to convert the optimal control problem into a parameter optimization problem, then using the Non-Linear Optimization (NLP) to solve the problem. In this paper, we use the method that directly targeting the parametric to convert the optimal control problem into the NLP problem. Firstly, discretize the time $t_{k}=t_{k}^{0}<t_{k}^{1}<\ldots .<t_{k}^{n}=t_{k}+T$, and parameterize the controlled quantity $u\left(t_{k}^{i}\right)=u_{i}, i=0, \ldots, N$. The NLP problem that converted is as follow.

$$
\begin{array}{cc}
F\left(u_{0}, \ldots, u_{N-1}\right)=\sum_{i=0}^{N-1} L\left(\bar{x}_{i}, u_{i}, t_{k}^{i}\right)\left(t_{k}^{i+1}-t_{k}^{i}\right)+V\left(\bar{x}_{N}, t_{k}^{N}\right) \\
\text { s.t. } \quad g\left(\bar{x}_{i}, u_{i}, u_{i}\right) \leq 0, i=0, \ldots, N \\
-\quad h\left(x_{N}\right)=0
\end{array}
$$

Then we give the control vector $u_{i}$ and initial state $\overline{x_{i}}\left(\overline{x_{i}}\left(\overline{x_{0}}=x_{k}\right)\right)$, the system state $x_{i+1}^{-}$of $t_{k}^{i+1}$ can be got by the step time integral of state equation, the SNOPT toolbox is used to solve the problem.

\section{The Flow Chart of the Multi-UAV Cooperative Air Combat Task Allocation}

The flow chart of the multi-UAV cooperative air combat task allocation is expressed as Figure 2. 


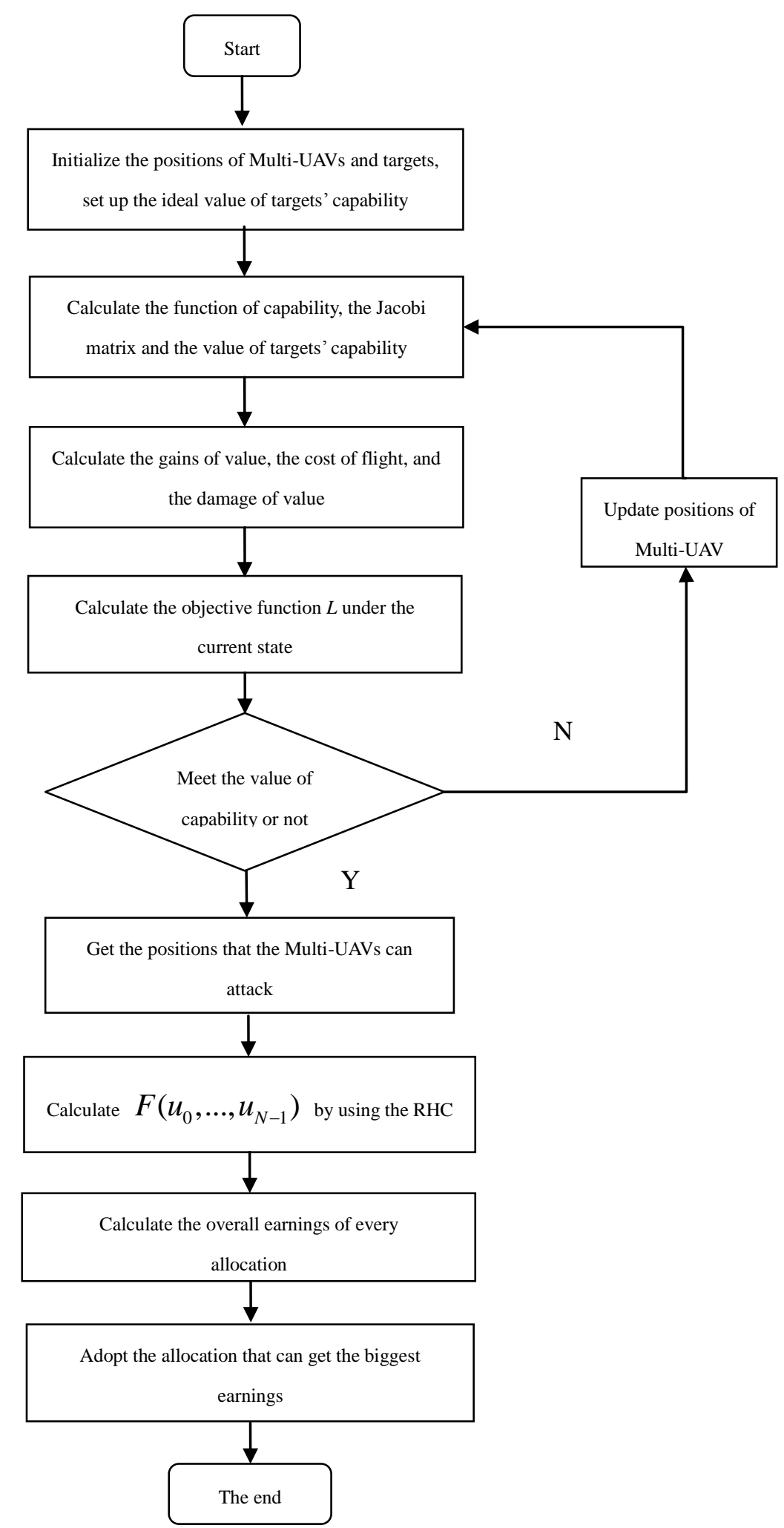

Figure 2. Flow Chart of the Task Allocation 


\section{Simulation Results and Analysis}

In this paper, four UAVs to attack two targets of the ground. Assuming that the number of weapon that the UAV carried is $\left[\begin{array}{llll}1 & 1 & 1 & 1\end{array}\right]$, and the number of anti-aircraft fire that every target has is one. The effective firing range of UAV is $12 \mathrm{~km}$ and the effective firing range of the target is $10 \mathrm{~km}$. The destroy effectiveness matrix of UAVs to targets is [0.95 0.940 .960 .93 ], and the destroy effectiveness matrix of targets to UAVs is [0.95 0.96].Besides,

$$
P_{\mathrm{UAV}}=\left[\begin{array}{lll}
3.78 & 4.28 & 4.26 \\
3.76 & 4.17 & 4.38 \\
3.83 & 4.22 & 4.28 \\
3.79 & 4.18 & 4.36
\end{array}\right](\mathrm{km}) \quad P_{\text {target }}=\left[\begin{array}{ccc}
0.95 & 0.52 & 0.53 \\
0.93 & 0.55 & 0.51
\end{array}\right](\mathrm{km}),\left[\begin{array}{l}
B_{1}^{d} \\
B_{2}^{d}
\end{array}\right]=\left[\begin{array}{c}
1.5 \\
1.5
\end{array}\right] .
$$

There are six allocation schemes for four UAVs to attack two targets. The allocation plans are shown as the Table 1 .

Table 1. Allocation Plans

\begin{tabular}{|l|l|l|l|l|l|l|}
\hline The plans & Plan 1 & Plan 2 & Plan 3 & Plan 4 & Plan 5 & Plan 6 \\
\hline $\begin{array}{l}\text { The allocation } \\
\text { matrix }\end{array}$ & {$\left[\begin{array}{llll}1 & 2 & 3 & 4\end{array}\right]$} & {$\left[\begin{array}{llll}1 & 3 & 2 & 4\end{array}\right]$} & {$\left[\begin{array}{llll}1 & 4 & 2 & 3\end{array}\right]$} & {$\left[\begin{array}{llll}2 & 3 & 1 & 4\end{array}\right]$} & {$\left[\begin{array}{llll}2 & 4 & 1 & 3\end{array}\right]$} & {$\left[\begin{array}{llll}3 & 4 & 1 & 2\end{array}\right]$} \\
\hline
\end{tabular}

The allocation matrix of plan 1 is $\left[\begin{array}{llll}1 & 2 & 3 & 4\end{array}\right]$. The matrix $\left[\begin{array}{llll}1 & 2 & 3 & 4\end{array}\right]$ means that the target 1 is attacked by the UAV 1 and UAV 2 , and the target 2 is attacked by UAV 3 and UAV 4. The following are similar.

Now, we allocate the UAVs by two methods which are as follow.

The method 1: To get the capability function of our UAVs, the Jacobian matrix, according to (2.3) and (2.4). Then calculate the gains of value, the cost of flight and the damage price according to (3.4) (3.7) and (3.8). At last we can get the earning value of every plan by using (4.2).The earning values are shown as Table 2.

Table 2. Earning Values of the Allocation Plans of Method 1

\begin{tabular}{|l|l|l|l|l|l|l|}
\hline The plan & Plan 1 & Plan 2 & Plan 3 & Plan 4 & Plan 5 & Plan 6 \\
\hline The earning value & 0.7338 & 0.7337 & 0.7109 & 0.7381 & 0.7111 & 0.7108 \\
\hline
\end{tabular}

We can see from the Table 2 that the biggest earning value belongs to the plan 4,in which the allocation is $\left[\begin{array}{llll}2 & 3 & 1 & 4\end{array}\right]$ means the target 1 is attacked by the UAV 2 and UAV 3, and the target 2 is attacked by UAV 1 and UAV 4, and the earning value is 0.7381 . It can be seen from the simulation that the smaller the controlled quantity and the Change in position of UAV, and the longer the simulation time as the capability value closer to the ideal value 2 . However, the damage effect can be got when the capability value reach 1.5 . So the capability value 1.5 can be regarded as the termination of the simulation condition. The capability that got by target 1 and target 2 in plan 4 is showed as Figure 3. 


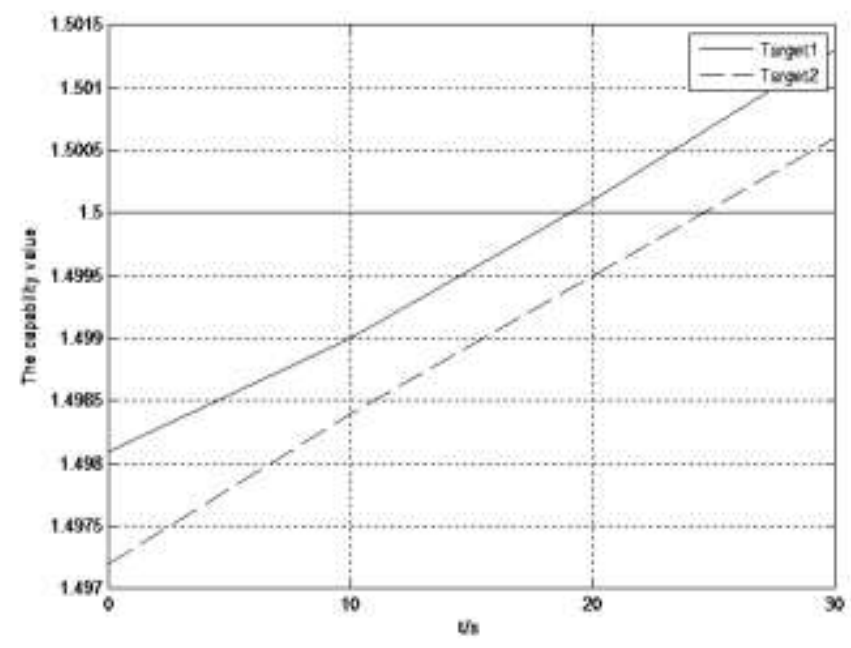

Figure 3. Capability Got By Target 1 and Target 2 In Plan 4

We can see from Figure 3 that the capability value is calculated once every 10 seconds, the capability of target 1 can reach 1.5 after two times, and the capability of target 2 can reach 1.5 after three times.

The method 2: According to the previous method of multi-UAV task allocation ${ }^{[12]}$, the capability function of multi-UAV cooperative attack and the capability that the targets needed are not considered, and the RHC is not used. In (3.10), $d_{i j}$ is the initial distance between the $i$-th UAV and the $j$-th target. Finally, we can get the earning value of every plan by simulation, and the earning value of every plan is shown as Table 3 .

Table 3. Earning Values of the Allocation Plans of Method 2

\begin{tabular}{|l|l|l|l|l|l|l|}
\hline The plan & Plan 1 & Plan 2 & Plan 3 & Plan 4 & Plan 5 & Plan 6 \\
\hline The earning value & 0.1037 & 0.1033 & 0.1036 & 0.1032 & 0.1038 & 0.1034 \\
\hline
\end{tabular}

We can see from the Table 6.3 that the biggest earning value belongs to the plan 5 , in which the allocation is $\left[\begin{array}{llll}2 & 4 & 1 & 3\end{array}\right]$ means that the target 1 is attacked by the UAV 2 and UAV 4, and the target 2 is attacked by UAV 1 and UAV 3, and the earning value is 0.1038 .

As can be seen from the results of the two methods, the difference between the two methods mainly includes three aspects. Firstly, the results of the two methods are different completely, that the allocation plan of the method 1 is plan 4 and the allocation plan of the method 2 is plan 5, and the earning value of method 1 is bigger than the other. Secondly, the capability of multi-UAV cooperative attack and the capability that the targets needed are not considered in the method 2. Therefore, we can draw the following conclusions that the method 1 can get more profit and damage efficiency of the targets, and improve the capability of multi-UAV cooperative attack.

\section{Conclusion}

This paper presents a new method for multi-UAV cooperative combat task allocation through the establishment of the capability function and Jacobian matrix. Then we use RHC to model the maneuvering decision making problem as the optimal control problem, and the optimal decision-making method of multi-UAV cooperative combat task allocation is gotten by the discretization of time and space in the whole process of decision-making and the calculation of the optimal maneuver strategy piecewise. The 
result of simulation shows that the method that considers multi-UAV cooperative task allocation capability can get more profit and damage efficiency of the targets, and improves the capability of multi-UAV cooperative combat compared with the multi-UAV cooperative task allocation capability is not considered, and it's a new method for multi-UAVs task allocation.

\section{Reference}

[1] Z.X. Yao, M. Li and Z.J. Chen, "Multi-agent framework of mission decision-making method for multi-aircraft cooperative combat", Electronics Optics \&Control, vol. 15, no. 3, (2008), pp. 1 4.

[2] L. B. Johnson, S. S. Ponda, H.-L. Choi and J. P. How, "Asynchronous Decentralized Task Allocation for Dynamic Environments", AIAA Guidance, Navigation, and Control Conference (GNC), (2011) (AIAA 2011-1441).

[3] T. Long, L.C. Shen, H.Y. Zhu and Y.F. Niu, "Distributed Allocation \& Coordination Technique of Multiple UAVs for Cooperative Tasks", ACTH AUTOMATICA SINICA, vol. 33, no. 7, (2007), pp. 731-737.

[4] Y. Liu, W.M. Li and Q.H. Xing, "Cooperative mission assignment optimization of unmanned combat aerial vehicles based on bilevel programming", Systems Engineering and Electronics, (2010), vol. 32, no. 3 , pp. 579-584.

[5] T.T. Wei and X.J. Qu, "Route planning method for multiple vehicles coordinated target assignment", Journal of Beijing University of Aeronautics and Astronautics, vol. 35, no. 8, (2009), pp. 917-924.

[6] J.Y. Du, F.M. Zhang, J.Yang and H.S. Wu, "Cooperative task assignment for multiple UCAV using particle swarm optimization", Control and Decision, vol. 27, no. 11, (2012), pp.1751-1755.

[7] J.W. Zhao, J.J. Zhao and W.B. Liu, "Multi-UAV Cooperative Task Allocation of Multi-task Air-to-ground Attack", Tactical Missile Technology, vol. 6, no. 38, (2014), pp. 38-44.

[8] X. Chen, X.M. Wei and G.Y. Xu, "Multiple Unmanned Aerial Vehicle Decentralized Cooperative Ai Combat Decision Making with Fuzzy Situation”, Journal Of Shanghai Jiao Tong University, (2014), vol. 48, no. 7, pp. 907-913.

[9] H.J. Jia, M. Xian and Y.G. Chen, “An Analysis of NCW and Its New Technology”, National Defense Science and Technology, vol. 32, no. 4, (2011), pp. 44-49.

[10] J. P. Chen, Z. G. Yuan and Y. M. Yang, "Multi-robot task allocation approach based on robotic utility function", Application Research of Computers, vol. 27, no. 4, (2010), pp. 1339-1441.

[11] P. Li, Y. M. Yang and J. L. Lian, "Utility-based task allocation for multi-robot system", Application Research of Computers, vol. 26, no. 2, (2009), pp. 537-539.

[12] X. Chen, Y. X. Hu and G. Y. Xu, "Two-dimensional Code PSO Algorithm for Multi-UCAV Task Assignment Problem", Journal of System Simulation, vol. 25, no. 12, (2013), pp. 3045-3049.

\section{Authors}
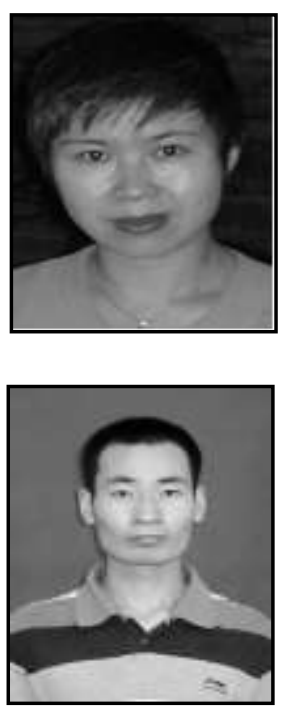

Xia Chen, she was born in 1962, she is a professor in Shenyang Aerospace University. Her main research interests are measurement technique, automatic control, flight control, etc.

Fuyu Zhao, he was born in 1989, he is a graduate student in Shenyang Aerospace University. His main research interest is the task allocation of unmanned aerial vehicle (UAV). 


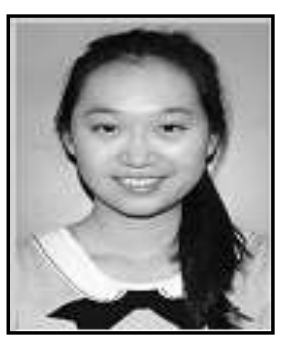

Liang Zhao, she was born in 1989, she is a graduate student in Shenyang Aerospace University .Her main research interest is the research of unmanned aerial vehicle (UAV) game theory. 
International Journal of Hybrid Information Technology

Vol. 10, No.1 (2017) 